\title{
Probing white-matter microstructure with higher-order diffusion tensors and susceptibility tensor MRI
}

\author{
Chunlei Liu ${ }^{1,2 *}$, Nicole E. Murphy ${ }^{1}$ and Wei Li ${ }^{1}$ \\ Brain Imaging and Analysis Center, School of Medicine, Duke University, Durham, NC, USA \\ 2 Department of Radiology, Duke University, Durham, NC, USA
}

\section{Edited by:}

Alpay Özcan, Virginia Polytechnic

and State University, USA

Reviewed by:

Jongho Lee, University of

Pennsylvania, USA

Pascal Sati, National Institutes of Health, USA

Yong Wang, Washington University in St. Louis, USA

\section{*Correspondence:}

Chunlei Liu, Brain Imaging and Analysis Center, School of Medicine, Duke University, 2424 Erwin Road, Suite 501, Durham, NC 27705, USA. e-mail: chunlei.liu@duke.edu
Diffusion MRI has become an invaluable tool for studying white matter microstructure and brain connectivity. The emergence of quantitative susceptibility mapping and susceptibility tensor imaging (STI) has provided another unique tool for assessing the structure of white matter. In the highly ordered white matter structure, diffusion MRI measures hindered water mobility induced by various tissue and cell membranes, while susceptibility sensitizes to the molecular composition and axonal arrangement. Integrating these two methods may produce new insights into the complex physiology of white matter. In this study, we investigated the relationship between diffusion and magnetic susceptibility in the white matter. Experiments were conducted on phantoms and human brains in vivo. Diffusion properties were quantified with the diffusion tensor model and also with the higher order tensor model based on the cumulant expansion. Frequency shift and susceptibility tensor were measured with quantitative susceptibility mapping and susceptibility tensor imaging. These diffusion and susceptibility quantities were compared and correlated in regions of single fiber bundles and regions of multiple fiber orientations. Relationships were established with similarities and differences identified. It is believed that diffusion MRI and susceptibility MRI provide complementary information of the microstructure of white matter. Together, they allow a more complete assessment of healthy and diseased brains.

Keywords: MRI, white matter, diffusion tensor imaging, generalized diffusion tensor imaging, susceptibility tensor imaging, higher order tensor, cumulant, kurtosis

\section{INTRODUCTION}

Probing the microstructure of white matter has major applications in a number of neurological diseases and disorders. The abilities to map brain connectivity in vivo were initially made possible because of the discovery of diffusion anisotropy in the white matter (Moseley et al., 1990) and the development of a range of techniques based on this phenomenon, most notably the development of diffusion tensor imaging (DTI) and tractography (Basser et al., 1994, 2000; Conturo et al., 1999; Mori et al., 1999). It is often reported that DTI, or more generally diffusion MRI, is the only method available for imaging white matter fiber tracts in vivo and non-invasively, which is a clear testament to the importance of DTI for studying brain connectivity. The recent discovery of magnetic susceptibility anisotropy (MSA) in white matter and the development of susceptibility tensor imaging (STI) may potentially provide a viable complementary method for imaging fiber tracts in vivo and non-invasively (Lee et al., 2010; Liu, 2010; Li et al., 2011, 2012a,b; Liu et al., 2012).

The existence of MSA has now been verified in simulation, mouse brains, specimens, and live human brains. For example, by rotating intact mouse brains in small-bore animal $7 \mathrm{~T}$ scanner, Liu showed that the magnetic susceptibility of white matter demonstrated strong orientation dependence (Liu, 2010). This orientation dependence has also been observed by Lee et al. on segments of corpus callosums from a postmortem human brain
(Lee et al., 2010). Similar to diffusion anisotropy, susceptibility anisotropy can also be described by a rank-2 tensor, the apparent susceptibility tensor (Liu, 2010). This susceptibility anisotropy and susceptibility tensor can be measured based on a simple 3D gradient-recalled-echo sequence. Variations among tissue magnetic susceptibility cause a tissue dependent frequency shift that manifests as a phase shift in gradient echo images. By measuring this frequency shift, a susceptibility tensor may be determined for each voxel of the brain. Susceptibility anisotropy and susceptibility tensors have thus far been imaged on both $3 \mathrm{~T}$ and $7 \mathrm{~T}$ in vivo (Li et al., 2011, 2012b; Schweser et al., 2012). Studies have shown that the major eigenvector orientation of the susceptibility tensor is aligned with axons in parallel (Lee et al., 2010; Liu, 2010; Li et al., 2012a,b; Liu et al., 2012). It has been reported that the apparent magnetic susceptibility (AMS) of the white matter is the most paramagnetic when the underlying axons are parallel to the magnetic field (Lee et al., 2010; Liu, 2010). It was proposed that this characteristic originates from the radially aligned myelin lipids (Li et al., 2012a). A recent study has utilized this relationship to perform fiber tracking in the mouse brain which has demonstrated similar tracks with DTI tractography in large fiber bundles (Liu et al., 2012).

An important challenge of MRI-based in vivo fiber tracking is the relative large voxel size (on the order of millimeters) compared to the size of axons which is on the order of micrometers. In large 
parallel fiber bundles, this mismatch of spatial scales is not problematic as the orientations of the axons are largely similar. However, in a large portion of the brain, each voxel of MRI images is known to contain fibers of different orientations, thus creating the situation of "crossing" or "kissing" fibers (Basser et al., 2000). Achieving high angular resolution by resolving these crossing fibers has been a major goal of diffusion based fiber tractography in the past decade (Frank, 2001, 2002; Alexander et al., 2002; Tuch et al., 2002; Lin et al., 2003; Liu et al., 2003a,b; Ozarslan and Mareci, 2003; Tuch, 2004; Jensen et al., 2005). A number of techniques have been proposed including, for example, q-space or diffusion spectrum imaging (King et al., 1994; Lin et al., 2003; Wedeen et al., 2008), q-ball imaging (Tuch, 2004) and higher order tensor models (Liu et al., 2003b, 2004; Ozarslan and Mareci, 2003) to name a few. Similarly, STI may encounter the same challenges. The behavior of magnetic susceptibility in the presence of these complex fiber architectures, however, has not been studied.

The goal of the present study was to investigate the behavior of magnetic susceptibility in the presence of fiber crossings and its relationship with higher order diffusion anisotropy. We first evaluated the variation of magnetic susceptibility as a function of magnetic field orientation in a simulated phantom of crossing fibers. The behavior was compared to that of higher order diffusion tensors calculated based on the cumulant expansion (covariance, skewness and kurtosis etc) (Liu et al., 2003a,b, 2004; Jensen et al., 2005). Experiments were also conducted in vivo to assess the effect of fiber crossings on magnetic susceptibility.

\section{MATERIALS AND METHODS SUSCEPTIBILITY TENSOR AND SUSCEPTIBILITY ORIENTATION DISTRIBUTION}

Since the early 1990s, diffusion MRI based on diffusion anisotropy has been the only means to study the orientations and organizations of white matter fiber tracts in vivo and noninvasively. In the past couple of years, it has become apparent that magnetic susceptibility of the white matter also exhibits anisotropy. While the ordered arrangement of axons largely contributed diffusion anisotropy (Beaulieu, 2002; Song et al., 2002), the ordered arrangement of myelin lipids is believed to be the main source of MSA (Li et al., 2012a). Susceptibility anisotropy may be quantified with the technique of susceptibility tensor imaging. Magnetic susceptibility of the brain results in a measurable frequency shift in the gradient-echo images. By solving the Maxwell's equation, this frequency shift denoted as $\theta$ was found to be related to a spatially distributed susceptibility tensor field $\chi(\mathbf{r})$ following (Liu, 2010)

$$
\theta=F T^{-1}\left\{\frac{1}{3} \hat{\mathbf{H}}^{\mathbf{T}} F T\{\mathbf{\chi}\} \hat{\mathbf{H}}-\hat{\mathbf{H}} \cdot \mathbf{k} \frac{\mathbf{k}^{\mathbf{T}} F T\{\mathbf{\chi}\} \hat{\mathbf{H}}}{k^{2}}\right\} \gamma H t
$$

Here, FT and $F T^{-1}$ represent Fourier and inverse Fourier transform respectively; $\mathbf{k}$ is the spatial frequency vector reciprocal to $\mathbf{r}$; $H$ is the magnitude of the applied magnetic field; $\hat{\mathbf{H}}$ is the unit vector of the applied magnetic field; $t$ is the echo time (TE) in a gradient echo sequence and $\gamma$ is the gyromagnetic ratio.
The susceptibility tensor $\chi(\mathbf{r})$ is assumed to be a $3 \times 3$ symmetric matrix with 6 independent elements ( 3 diagonal and 3 off diagonal). When the demagnetization field [the second term of Equation (1)] is ignored, this symmetry property is evident as swapping the indices of $\chi(\mathbf{r})$ does not change $\theta$. Given this symmetry assumption, a minimum of 6 independent measurements are needed to determine a susceptibility tensor. Each independent measurement will require the magnetic field to be oriented in a different direction with respect to the object. As an analogy to DTI, this is equivalent to changing the directions of diffusion encoding gradients. And, similar to the measurement of the diffusion tensor, the angle separation between orientations should ideally spread apart. As in any other experimental measurements, more orientations will improve the matrix condition and allow a more accurate determination of the tensor elements. Once the susceptibility tensor is calculated, it can then be decomposed into its eigensystem with the eigenvalue decomposition. One advantage of eigenvalue decomposition is that it expresses susceptibility tensor in a coordinate system that is independent of the experimental coordinate system. As a result, the values can be compared between different scans and different subjects.

The relationship between susceptibility tensor and fiber orientations has been established in the case of parallel fibers. It has been shown that the direction of the major eigenvector of the susceptibility tensor (most paramagnetic) is parallel to the direction of the axons (Lee et al., 2010; Liu, 2010). The relationship has been confirmed broadly with theory, simulation, brain specimens, ex vivo and in vivo brain imaging at $3 \mathrm{~T}$ and $7 \mathrm{~T}$. In the case of fiber crossing, however, a single susceptibility tensor is unlikely to convey the existence of multiple fiber orientations. One way to address this limitation is to use multiple susceptibility tensors to characterize the susceptibility property of a voxel. Alternatively, we can plot the AMS as a function of the field orientation. The orientation variation of the AMS may depict the existence of multiple fibers. These orientations can then be compared to those based on diffusion models.

\section{HIGHER-ORDER TENSOR (HOT) RECONSTRUCTION}

In the cumulant expansion, the diffusion-weighted MRI signal is related to a set of higher-order tensors following (Liu et al., 2003b, 2004)

$$
s(b)=s(0) \exp \left\{\sum_{n=2}^{\infty}(-j)^{n} D_{i_{1}, i_{2}, \ldots, i_{n}}^{(n)} b_{i_{1}, i_{2}, \ldots, i_{n}}^{(n)}\right\}
$$

Here, $s(b)$ is the diffusion weighted signal and $s(0)$ is nondiffusion weighted signal; $j$ is the imaginary number; $D^{(n)}$ is the $n$-th order cumulant tensor with $n=2$ corresponding to the covariance matrix, $n=3$ corresponding to the skewness tensor and $n=4$ corresponding to the kurtosis tensor; $b(n)$ is an $n$-th order tensor describing diffusion weighting factors. The Einstein's summation rule is assumed for the subscripts of $D^{(n)}$ and $b^{(n)}$. It has been shown that $D^{(n)}$ is a symmetric tensor as it is related to the partial differentiation of the particle concentration in Fick's second law (Liu et al., 2003b, 2004).

Although Equation (2) can be readily solved with least-squares estimations given a set of measurements at different diffusion 
encoding directions and $b$-values, the solution for the higherorder tensor (HOT) may be easily biased by the noise at large $b$ values. Specifically, because $s(b)$ is Rician distributed, it does not decay to zero. Instead, it approaches the noise floor as the $b$-value increases, resulting in an overestimate of the non-Gaussianity and higher-order tensors. To reduce this overestimation, we apply Tikhonov regularization by solving the following minimization problem for a fourth-order approximation

$$
\begin{gathered}
\min _{D^{(2)}, D^{(4)}}\left\|\ln (s(b) / s(0))+b_{i_{1}, i_{2}}^{(2)} D_{i_{1}, i_{2}}^{(2)}-b_{i_{1}, i_{2}, i_{3}, i_{4}}^{(4)} D_{i_{1}, i_{2}, i_{3}, i_{4}}^{(4)}\right\|^{2} \\
+\left\|\Gamma_{i_{1}, i_{2}, i_{3}, i_{4}} D_{i_{1}, i_{2}, i_{3}, i_{4}}^{(4)}\right\|^{2}
\end{gathered}
$$

The Tikhonov matrix $\Gamma$ is a diagonal fourth-order tensor with a diagonal element of

$$
\lambda=\operatorname{trace}\left(b^{(4)}\right)\left(\bar{D} / D_{0}\right)^{5}
$$

Here, $\bar{D}$ is the mean diffusivity of a given voxel estimated by DTI using the smallest $b$-value; $D_{0}$ is a diffusion coefficient chosen to be $1.5 \times 10^{-3} \mathrm{~mm}^{2} / \mathrm{s}$, slightly smaller than that of cerebral spinal fluid but larger than that of gray matter. With this choice of regularization parameter, the fourth-order tensor of the CSF is suppressed assuming the diffusion is mainly Gaussian in the CSF. By raising the ratio of $\bar{D}$ and $D_{0}$ to the fifth power, the effect of regularization is reduced for the white matter. The scaling factor of trace $\left[b^{(4)}\right]$ is included to ensure the regularization term is dimensionless.

Coordinate system independent quantities can be defined with eigenvalue decomposition. There have been some increasing efforts to better understand the algebra of fourth-order tensors in the past decade (Miehe, 1993; Browaeys and Chevrot, 2004; Muti and Bourennane, 2005). Nevertheless, a standard of decomposition for fourth-order tensors is not fully established yet. We chose to convert the fourth-order diffusion tensor with a dimension of $3 \times 3 \times 3 \times 3$ to a two-dimensional $9 \times 9$ matrix denoted as $\mathbf{T}$. This technique has been widely used in describing the stiffness tensor of materials' mechanical property (Nemat-Nasser and Hori, 1999; Basser and Pajevic, 2007). This can be accomplished by transferring each $D_{i_{1} i_{2} i_{3} i_{4}}^{(4)}$ component to element $T_{\mathrm{pq}}$. The rule of transformation between $4 \mathrm{D}$ index and $2 \mathrm{D}$ index is given in Table 1. For example, the fourth-order element $D_{1231}$ is transformed to element $T_{27}$ following the rule. Decomposing tensor $\mathrm{T}$ results in 9 eigenvalues.

Once the higher order diffusion tensors are measured, the probability density function (PDF) of the underlying diffusion process can be computed using the Gram-Charlier series (Liu et al., 2003b, 2004). The Gram-Charlier series is widely used

Table 1 | Indices for unwrapping a fourth-order tensor $D_{i j k l}$ into a $9 \times 9$ matrix $T_{p q}$.

\begin{tabular}{llllllllll}
\hline$p(q)$ & 1 & 2 & 3 & 4 & 5 & 6 & 7 & 8 & 9 \\
$i j(k /)$ & 11 & 12 & 13 & 21 & 22 & 23 & 31 & 32 & 33
\end{tabular}

expansion based on the Hermite tensor (Zucker and Schulz, 1982).

\section{PHANTOM EXPERIMENTS AND SIMULATION}

A phantom of crossing fibers was used for diffusion experiments. The phantom consisted of sheets of parallel plastic capillaries with an inner diameter of $50 \mu \mathrm{m}$ and an outer diameter of $350 \mu \mathrm{m}$ (PTFE ultramicrobore tubing P-06417-70, Cole-Parmer Instrument, Vernon Hills, IL). Two sets of capillaries were overlapped at a $90^{\circ}$ angle. Imaging was performed on a 3T MRI system with a high-performance insert gradient set. Scan parameters were: $\mathrm{FOV}=25 \times 25 \mathrm{~cm}^{2}, T R=1900 \mathrm{~ms}, T E=13.8 \mathrm{~ms}$, single slice, $N E X=4$ and matrix size $=32 \times 32$. Diffusion-weighted images were acquired with a stimulated echo for 160 diffusion encoding directions and for $b$-values of 500, 1000, 2000, 4000, and $8000 \mathrm{~s} / \mathrm{mm}^{2}$.

However, this phantom did not mimic the susceptibility effect of myelin structures while it is known that myelin is the primary origin of susceptibility anisotropy. Secondly, the bulk susceptibility effect was also a concern in part due to imperfect elimination of air bubbles in this type of phantoms. To control these effects, we constructed a numerical phantom that simulates the structures of packed axons in the white matter more precisely. Specifically, a cubic voxel packed with an ensemble of parallel axons was generated. The voxel had a dimension of $d=256 \mu \mathrm{m}$ on all sides. The axons were aligned along the $z$-axis. The inner radius of the axon was $3.5 \mu \mathrm{m}$ and the outer radius was $5.0 \mu \mathrm{m}$. The distance between two neighboring axons was $11.0 \mu \mathrm{m}$. The susceptibility of the axons was set to be $-0.082 \mathrm{ppm}$ and the susceptibility anisotropy $\left(\chi_{\|}-\chi_{\perp}\right)$ of the myelin sheath was 0.163 ppm with $\chi_{\|}$being $-0.1 \mathrm{ppm}$ (Li et al., 2012a). Here $\chi_{\|}$ is defined as the susceptibility along the direction parallel to the axon. The susceptibility of the interstitial space was assumed to be zero as the reference. The voxel was divided into a $512 \times 512 \times$ 512 grid resulting in a grid size of $0.5 \mu \mathrm{m}$. A susceptibility tensor was assigned to each grid point depending on the tissue type (myelin, axon, or interstitial space). Only grid points within the myelin sheath had anisotropic tensors. The major eigenvectors of the myelin tensors were perpendicular to the long axis of the axon.

Once the susceptibility of each grid point within the voxel was assigned, the magnetic field at each grid point was computed via the forward Fourier relationship between susceptibility tensor and magnetic field as expressed in Equation (1). This computed magnetic field is what needed to satisfy Maxell's equations given the distribution of magnetic susceptibility and the presence of the external $\mathbf{B}_{0}$ field. The MR signal generated by the voxel was evaluated at $T E=20 \mathrm{~ms}$ and a field strength of 3T. Specifically, each grid point within the voxel (a total of $512^{3}$ grid points) was assumed to have the same proton density; however, each grid precessed at its own frequency corresponding to the magnetic field of its specific location. For a given external field orientation, the total signal of the voxel was computed as a complex summation of signals originating from all grid points within the voxel. A total of 10,000 field orientations were simulated. The mean frequency shift of the whole voxel was computed for each orientation by computing the phase of the summed signal. From this mean frequency shift, the AMS was 
computed by inverting Equation (1). Because only a single voxel was present, the second term in Equation (1), i.e., the demagnetization term was ignored when computing the AMS for each orientation.

\section{MRI EXPERIMENTS In vivo}

A healthy adult was scanned on a 3T MRI system (GE MR750, GE Healthcare, Waukesha, Wisconsin) equipped with an 8-channel head coil and a maximal gradient strength of $50 \mathrm{mT} / \mathrm{m}$. Diffusion weighted images were acquired using a dual-echo sequence and following parameters: $\mathrm{FOV}=256 \times 256 \mathrm{~cm}^{2}$, matrix size $=$ $128 \times 128$ (reconstructed to $256 \times 256$ by zero filling), $T R=$ $10.2 \mathrm{~s}, T E=98.5 \mathrm{~ms}$, number of slices $=73$, number of diffusion directions $=30$ and $b$-values of 1000 and $2500 \mathrm{~s} / \mathrm{mm}^{2}$. Five images with $b=0$ were also acquired. All images were acquired in a single run to maintain the same T2-weighting for both $b$-values.

Gradient-echo images were also acquired on the same scanner. A quadrature head coil was employed for the gradient echo imaging to allow a wider range of head orientations inside the coil. Gradient-echo images with various head orientations with respect to the main magnetic field were acquired using a standard flow-compensated 3D spoiled-gradient-recalled-echo (SPGR) sequence with the following parameters: $T E=40 \mathrm{~ms}$, $T R=60 \mathrm{~ms}$, flip angle $=20^{\circ}, \mathrm{FOV}=256 \times 256 \times 256 \mathrm{~mm}^{2}$, matrix size $=128 \times 128 \times 128$. Shimming was performed at each head orientation. A total of 16 orientations were acquired to achieve the rotation angle of $-42 \sim 52^{\circ}$ (around the anteriorposterior direction) and $-43 \sim 39^{\circ}$ (around the left-right direction). All human studies were approved by Institutional Review Board.

Image phase was unwrapped with a Laplacian-based phase unwrapping algorithm (Li et al., 2011; Wu et al., 2012) and filtered with the sphere mean value filtering, with a radius of 25 voxels with the radius decreasing toward the brain boundary (Li et al., 2011). Phase value was normalized by the TE to yield the frequency shift. AMS was first quantified at each brain orientation with respect to the main magnetic field using the algorithm for sparse linear equations and sparse least squares (LSQR) (Paige and Saunders, 1982). The multi-orientation gradient echo images were linearly registered to the non-diffusion weighted DTI images using FSL-FLIRT (Oxford Center for Functional MRI of the Brain, Oxford, UK). The resulting transformation matrix was used to rotate the phase and susceptibility maps and to determine the brain orientation with respect to the main magnetic field.

\section{RESULTS}

\section{HOT OF CROSSING-FIBER PHANTOM}

Figure 1 shows the geometry and signal behavior in the diffusion phantom. The phantom consisted of two sets of capillaries intersecting at $45^{\circ}$ as illustrated by the T2-weighted image (Figure 1A). Four voxels were selected within the phantom. One voxel situated in the intersecting area represented by a green box; a second voxel situated in an area with a single fiber orientation $\left(45^{\circ}\right)$ represented by a blue box; a third voxel situated in a area with vertically oriented fibers represented by a magenta box; a fourth voxel situated in the surrounding liquid represented by a red box (Figure 1A). The SNR at $b=0$ was 2536, 1134 , and 75 respectively for liquid, single fibers, and crossing fibers. The diffusion signal in these four voxels was plotted as a function of the $b$-value along the diffusion encoding direction of $[0,0,1]$ (Figure 1B). The signal curves were displayed in logarithmic scale and coded with the color as the voxels were labeled in Figure 1A. In the fluid, the signal decayed exponentially up to $b=2000 \mathrm{~s} / \mathrm{mm}^{2}$. At $b=4000 \mathrm{~s} / \mathrm{mm}^{2}$, the signal was approaching the noise level and the decay curve bent upward (arrow in Figure 1B), giving a false impression of non-Gaussian diffusion. Similar behavior was observed in the single fiber voxel. In the voxel of crossing fibers, the signal decay curve bent upward rather early at $b=1000 \mathrm{~s} / \mathrm{mm}^{2}$ while the signal strength was still more than 10 times higher than the noise level.

Figure 2 compares the higher order tensors estimated with and without Tikhonov regularization. Figures $\mathbf{2 A}, \mathbf{C}$ compares the

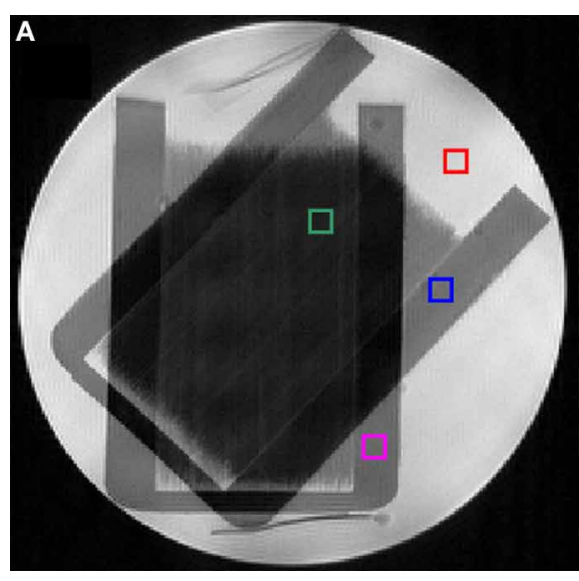

FIGURE 1 | Signal behavior of diffusion weighted images of a phantom. (A) A T2-weighted image shows the crossing fiber structure of the phantom. Four voxels highlighted regions of fiber crossing (green), single fiber in a $45^{\circ}$-angle (blue), vertical fiber (magenta) and isotropic fluid (red).

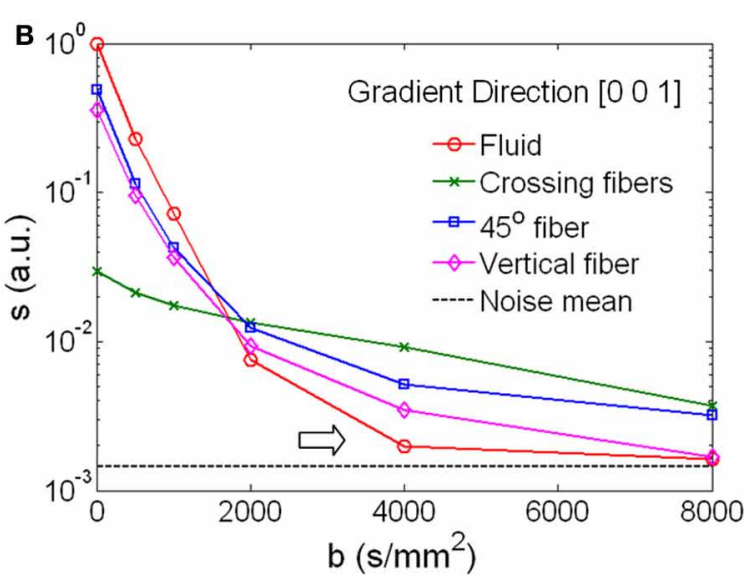

(B) Diffusion-weighted signals as a function of the $b$-values showed distinctive characteristics for the four voxels highlighted in (A). In the fluid and the single fiber along the direction of $[0,0,1]$, the signals approached the noise floor when $b>2000 \mathrm{~s} / \mathrm{mm}^{2}$ (arrow). 


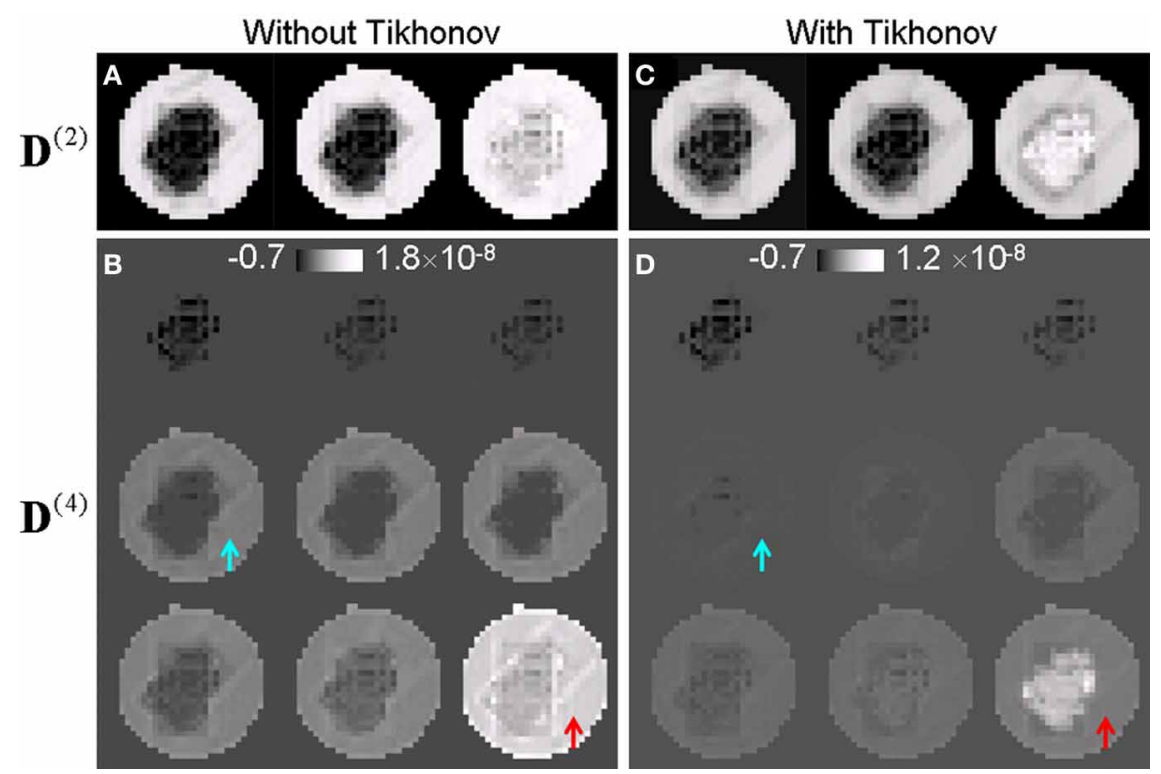

FIGURE 2 | Higher-order tensors estimated with and without Tikhonov regularization. $(\mathbf{A}, \mathbf{B})$ Eigenvalues of the second and the fourth order tensor estimated without regularization. The fourth order tensor was overestimated for the fluid (arrows). (C,D) Corresponding eigenvalues of the second and the fourth order tensor estimated with regularization. The contrast between regions of fiber crossing and surrounding areas was significantly improved. three eigenvalues of the second order tensor while Figures 2B,D compares the nine eigenvalues of the fourth order tensor. Without Tikhonov regularization, the fourth order tensor elements were severely overestimated in the liquid region. Without regularization, the eigenvalues of the fourth order tensor in the liquid was similar or slightly larger than those of the crossing fibers (Figure 2B) which is not physical as the diffusion in the liquid is expected to be largely Gaussian. The application of regularization suppressed the fourth order tensor in the liquid (arrows in Figure 2D). Note that the eigenvalues of the fourth order tensor can be negative as the tensor is not restricted to be positive definite in general. Similar results were observed in vivo. ${ }^{\mathrm{R} 3}$

\section{DIFFUSION PDF AND AMS ORIENTATION DISTRIBUTION IN CROSSING FIBERS}

Figure 3 compares the fiber crossing reconstructed by higherorder diffusion tensors and that by the apparent magnetic susceptibility. From the PDF glyphs, regions of fiber crossing and regions of single fibers were readily identifiable (Figure 3B). These regions matched exactly as shown in the anatomical T2-weighted image (Figure 3A). The crossing angle measured based on the PDF glyphs also agreed with the true crossing angle of $45^{\circ}$. AMS profile was evaluated on a simulated fiber crossing in a cubic voxel. Figure 3C illustrates the arrangement of axons within the voxel. Each fiber consists of an axon and insulating myelin sheath which has an anisotropic susceptibility tensor (Figure 3B). The orientation distribution of the calculated AMS demonstrated two components intersecting at $45^{\circ}$ consistent with the underlying geometry.

\section{HUMAN BRAIN in vivo}

The SNR of the diffusion-weighted brain images at $b=0$ was 60 for the parenchyma on average. The SNR of the gradientecho images was 34.8 for the parenchyma. Figure 4 illustrates the quality of fitting based on the susceptibility tensor model. The expected frequency maps based on the fitted susceptibility tensors are slightly smoothed but demonstrate similar contrast as the corresponding experimental frequency maps (Figure 4A). The rootmean-squared-error (RMSE) map for a representative orientation shows elevated error around tissue boundaries, but demonstrating small errors in most areas of the brain (Figure 4B). This consistency is further illustrated by three line profile plots (Figure 4C) and a correlation analysis (Figure 4D). Small deviations between experimental and fitted values were observed in the line profiles. Deviations within the white matter regions indicate potential inadequacy of the tensor model that may be induced by more complicated fiber structures. Overall, the correlation coefficient was high with $R^{2}=0.8833$.

Figure 5 shows the fiber orientations reconstructed by HOT and the corresponding behavior of AMS as a function of fiber angle determined by DTI. In the top row of Figure 5A, the PDF glyphs showed parallel fibers in a selected region of interest (ROI); in the bottom row of Figure 5A, the PDF glyphs of the selected ROI showed extensive fiber crossings. In the ROI with parallel fibers, AMS followed a sine-squared relationship with the fiber angle (top row of Figure 5B). The fiber angle was computed as the angle between the major eigenvector of the diffusion tensor and the $\mathrm{B}_{0}$ field. The principal orientations provided by STI showed resemblance to those by DTI in this region; however, significant differences also existed, consistent with previous reports (Li et al., 2012a,b). These discrepancies were thought to be caused 


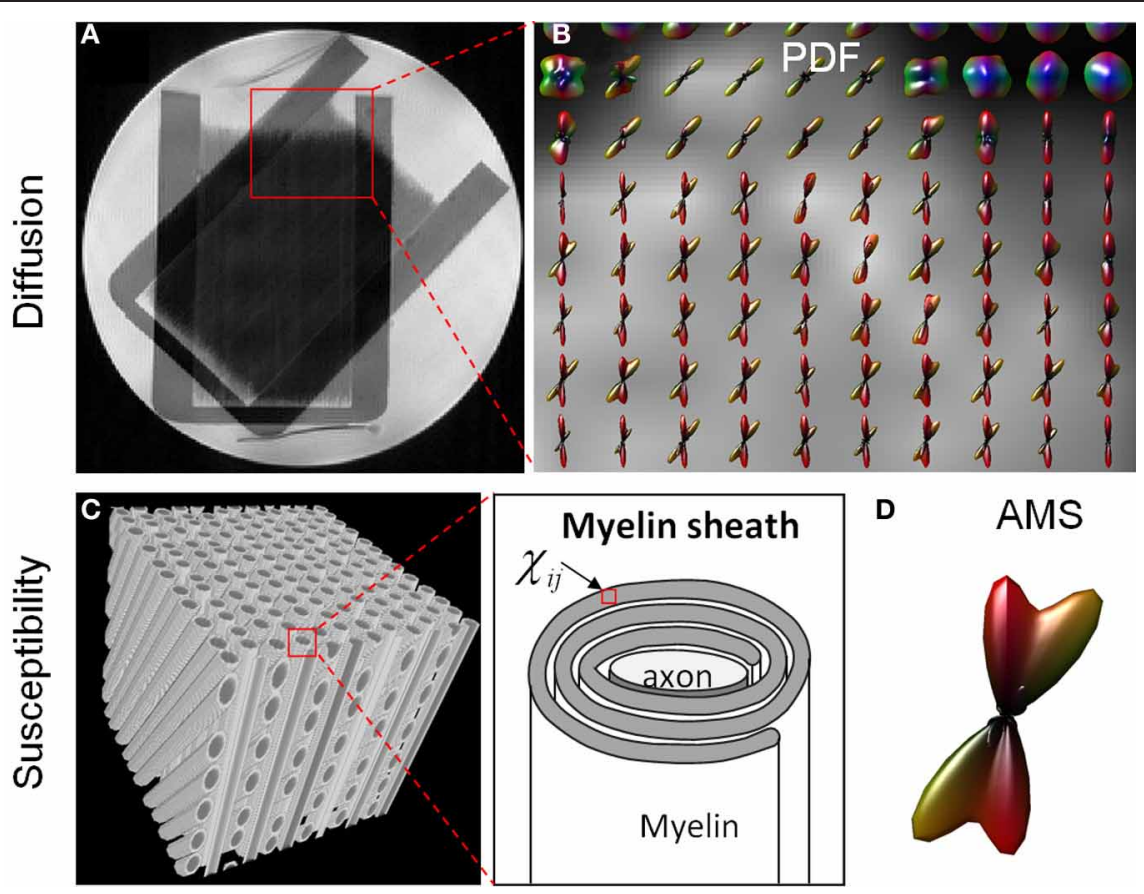

FIGURE 3 | Comparison of HOT (A,B) and AMS (C,D) glyphs in fiber crossing. (A) A T2-weighted image illustrated a region of complex fiber structures with both single fibers and crossing fibers (red box). (B) The PDF glyphs over this ROI reconstructed by HOT agreed with the known phantom structure. (C) An illustration of myelinated axons crossing at $45^{\circ}$. Each voxel in the myelin sheath contained an anisotropic susceptibility tensor. (D) The distribution of AMS depicted a $45^{\circ}$ crossing. by residual field gradients. ${ }^{R 3}$ On the other hand, in the ROI with fiber crossings, AMS no longer followed the sine-squared relationship (bottom row of Figure 5B).

\section{DISCUSSION}

The discovery of anisotropic diffusion has led to an explosive development of diffusion based MRI techniques for probing the microstructure of brain white matter and brain connectivity. Until very recently, diffusion MRI, in particular diffusion tensor MRI, had been only way capable of imaging white matter fiber orientations in vivo and non-invasively. The findings of anisotropic magnetic susceptibility in the white matter may eventually provide an alternative method. In this study, we explored for the first time the behavior of AMS in the case of fiber crossing. The behavior was investigated both in phantoms and in vivo. The relationship between complex AMS profile and non-Gaussian diffusion in these situations were evaluated based on the high-order tensor model of diffusion.

Previous studies have shown that in the case of parallel fibers, the orientation dependence of magnetic susceptibility can be described by a rank-2 tensor (Liu, 2010). The orientation of the major eigenvector of the susceptibility tensor was found to be aligned with the underlying fibers (Liu, 2010; Li et al., 2012a,b). Initial fiber tracking based on STI demonstrated similar tracts with DTI tractography in major white matter tracks of mouse brains ex vivo (Liu et al., 2012). However, it is also known that the eigenvector orientations of susceptibility tensors may not completely agree with those of diffusion tensors in vivo.
Part of the mismatch has been attributed to residual field gradients and imperfect image registration (Li et al., 2012a,b). Another important source of the mismatch could be the complex fiber architecture of white matter fibers that deviate from the simple model geometry of parallel axons. The current study extended the analysis to cases of crossing fibers. In the phantom of $45^{\circ}$ crossing, the angular distribution of AMS did not show an ellipsoidal shape. Rather, extremal AMS was found in directions along the fibers. In brain white matter of mainly parallel fibers such as the corpus callosum, AMS follow the typical sine-squared relationship as a function of fiber angles with respect to the main field. In regions of fiber crossing, AMS no longer followed this simple relationship. Although we were not able to illustrate the orientation distribution of AMS in 3D due to the limited number of orientations available, the observed deviation from the sine-squared relationship clearly indicated the existence of microstructures that differed from parallel fibers.

These behaviors of AMS are reminiscent of that of ADC in the situation of non-Gaussian diffusion. To explore whether the observed behavior of AMS indeed corresponded to the characteristics of crossing fibers, we reconstructed the underlying fibers with the higher order tensor model of diffusion. Diffusion tensors up to the fourth order were computed for both the phantom and the human brain. To control the bias caused by the nonzero noise floor in Rician distributed diffusion-weighted signals, we applied a Tikhonov regularization based on the mean diffusion coefficient. If the mean diffusion coefficient is larger than $1.5 \times 10^{-3} \mathrm{~mm}^{2} / \mathrm{s}$, then the corresponding higher order tensor 
A
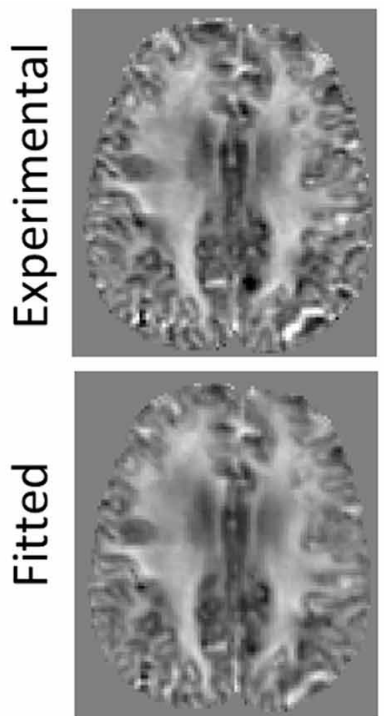
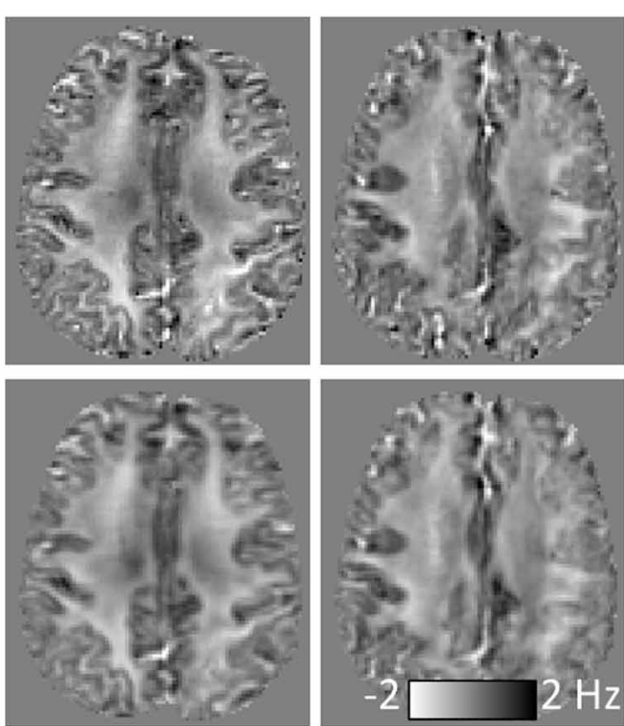

\section{RMSE}
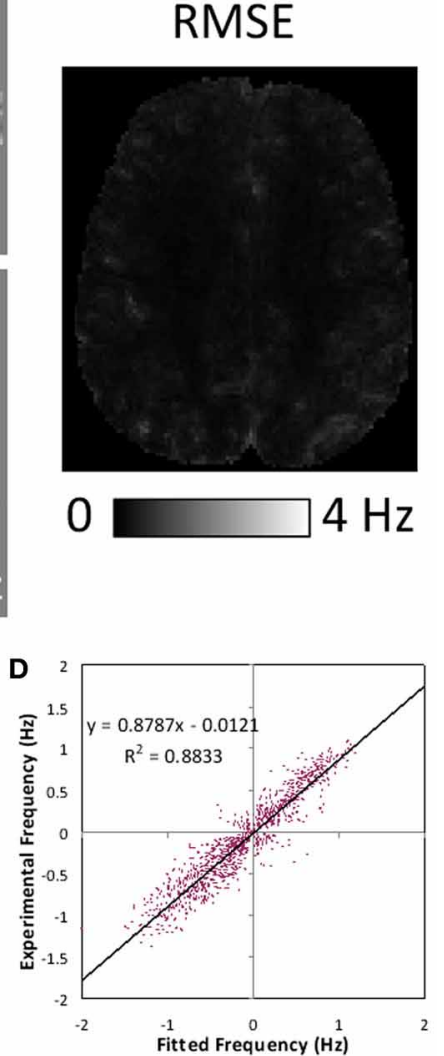

Fitted Frequency $(\mathrm{Hz})$
C

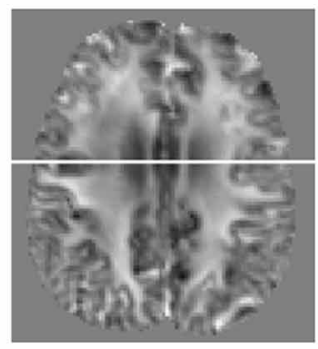

Line profiles of 3 orientations

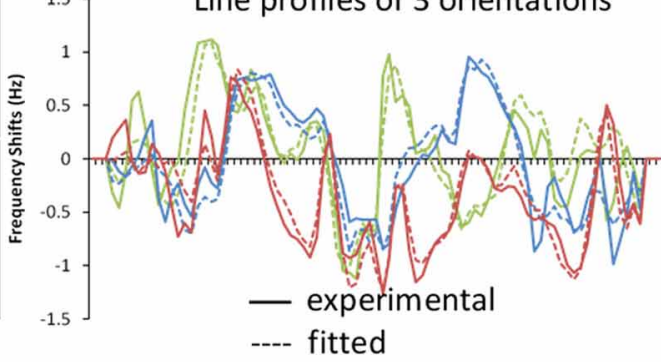

FIGURE 4 | Comparison of frequency maps measured experimentally and those fitted with susceptibility tensors. (A) Comparison of representative experimental and fitted frequency maps along three orientations. Fitted maps were slightly smoothed but showing similar contrast. (B) A representative root-mean-squared-error (RMSE) map between the experimental and fitted frequency along one orientation. Significant errors were only observed around tissue boundaries. (C) Line profiles of experimental and fitted frequency maps for the same three orientations as in (A). (D) Experimental and fitted frequency values along the line shown in (C) demonstrating high correlation. elements are penalized. This regularization effectively suppressed the otherwise artificially high higher order tensor elements in the liquid of the phantom and the CSF of the brain. In addition, the tensors of fiber crossings were more accurately estimated as evidenced by the enhanced contrast of areas of fiber crossings. Alternatively, the issue of Rician distributed signal may also be resolved with a maximum likelihood estimator once the statistics of the Rician signal is estimated (Sijbers and den Dekker, 2004; Basu et al., 2006). However, this alternative strategy is generally computationally expensive over a $3 \mathrm{D}$ volume of images. The Tikhonov approach was efficient without any significant increase of computational burden. The corresponding PDF reconstructed based on these tensors accurately matched the fiber crossing features in the phantom. In the human brain, the PDF showed single fiber orientations in the corpus callosum which explained the sine-squared behavior of AMS. Away from these simple structures, the corresponding PDF clearly illustrated the underlying fiber crossings which explained the deviation of AMS from the sine-squared behavior.
Our preliminary investigation of magnetic susceptibility behavior in crossing fibers indicated that magnetic susceptibility may provide sufficient information for resolving fiber crossings in certain cases. To fully capture the structural characteristics, it appeared that more orientational sampling would be necessary. This was demonstrated on a simulated voxel of crossing fibers. The complex signal of the voxel was evaluated at multiple field orientations. It was found that the orientation distribution of AMS was indicative of the fiber structure. The associated challenge obviously is the increased scan time and necessity to change the position of the brain with respect to the main magnetic field. For example, while we showed that AMS no longer follows a sine-squared relationship in vivo in the case of fiber crossing (Figure 5), the limited number of orientations did not allow us to plot the 3D distribution of AMS. With the available number of orientations, we were able to detect fiber crossing but not able to determine the multiple fiber orientations. ${ }^{\mathrm{R} 3}$ Currently, this challenge is substantial in vivo but the technique can be readily applied ex vivo or in some cases of small animal imaging. 


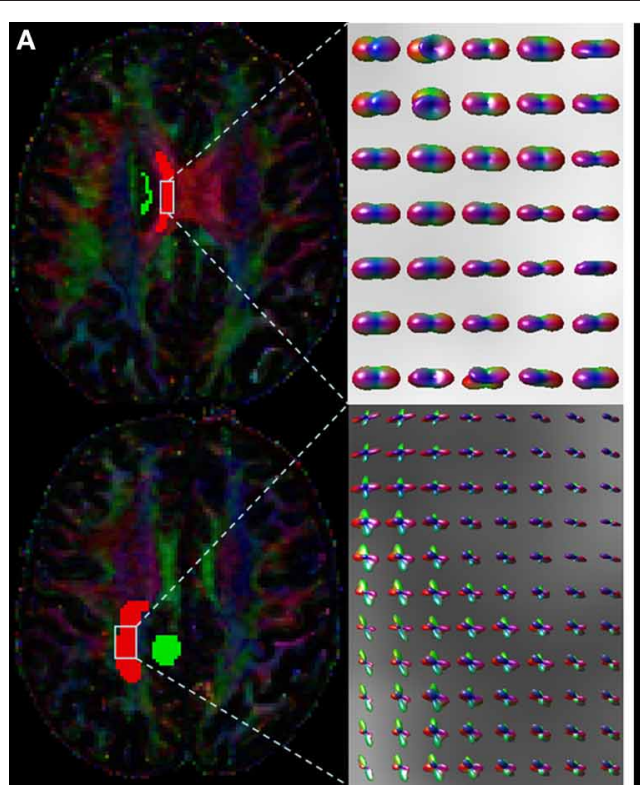

FIGURE 5 | Comparison of fiber orientations reconstructed by HOT and the orientation dependence of AMS. (A) Color coded FA map reconstructed by DTI (left) and fiber orientations of selected ROI reconstructed by HOT. The color scheme was: red representing left-right, green representing anterior-posterior and blue representing superior-inferior. In the top row, fiber orientations were primarily unidirectional in the left-right direction; in the bottom row, fiber
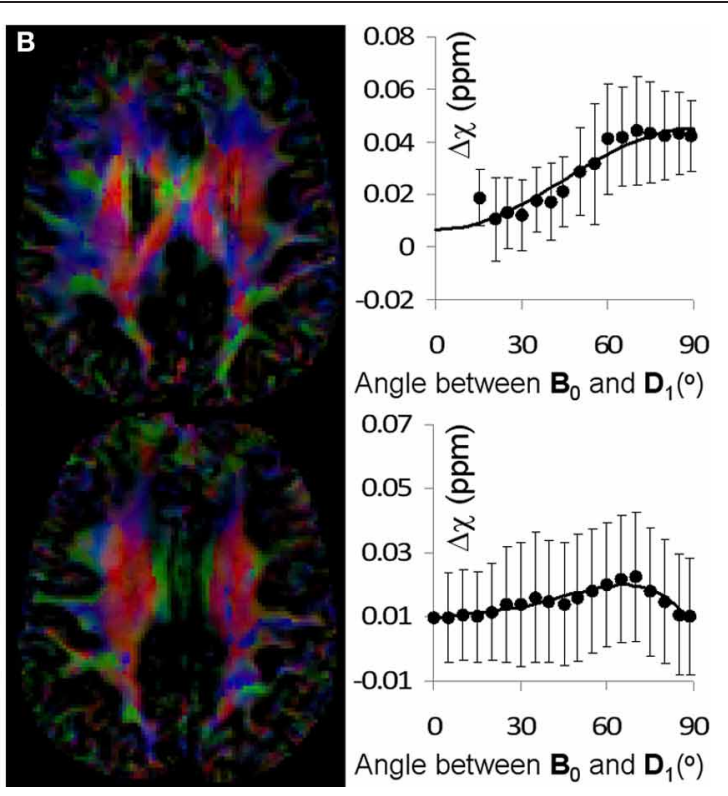

crossings were extensive and clearly visible in the PDF glyphs. (B) STI eigenvector orientation map (left) and corresponding AMS plot as a function of fiber angle over the same ROI in (A). AMS was calculated using the adjacent green $\mathrm{ROI}$ as a reference to reduce global heterogeneity of magnetic susceptibility. Fiber angles were computed as the angles between DTI fiber orientations and the $\mathrm{B}_{0}$ field (superior-inferior).
When a sufficient number of orientations are provided, the structural information may be decoded by analyzing the orientation distribution of the AMS. A simple graphic display of AMS in 3D may be sufficient to identify the underlying fiber orientations. More sophisticated or quantitative method may also be developed such as with the use of spherical harmonic functions, higher order tensors or the recently proposed multipole tensors ( $\mathrm{Liu}$ and Li, 2013).

Further studies are needed to determine whether combining susceptibility and diffusion information may provide a more complete characterization of tissue microstructure. It has been shown that susceptibility anisotropy originates from two properties of the white matter. One is the diamagnetic property of the lipid molecules of the myelin bilayer; the other is the ordered arrangement of the lipid molecules around the axons (Lee et al., 2010; Liu, 2010; Li et al., 2012a). On the other hand, diffusion anisotropy appeared to originate primarily from the axon itself. When the myelin is removed from the white matter, for example, in the shiverer mouse, diffusion anisotropy is reduced by about $10-15 \%$ while susceptibility contrast and susceptibility anisotropy of the white matter largely disappeared (Liu et al., 2011). The information provided by susceptibility and that by diffusion are thus complementary. Probing the microstructure of white matter simultaneously with susceptibility and diffusion imaging may be beneficial for differentiating abnormalities that are due to axon degeneration and demyelination. Susceptibility imaging may be advantageous in situations when high spatial resolution is required as the resolution of diffusion-weighted images has largely been limited to be around
$2 \times 2 \times 2 \mathrm{~mm}^{3}$. Susceptibility images, on the other hand, can be readily acquired at much higher spatial resolution and they are inherently three dimensional. Susceptibility contrast is also inherently advantageous at ultra-high field strength $(\geq 7 \mathrm{~T})$ due to increased phase contrast (Duyn et al., 2007), low specific absorption rate and minimal sensitivity to B1 field inhomogeneity. The application of diffusion weighted images at 7T has been significantly hampered by increased RF heating and B1 inhomogeneity. Integrating susceptibility and diffusion contrast at ultra-high field strength may therefore become especially beneficial.

In conclusion, we showed that magnetic susceptibility may be used to probe complex white matter structures in the presence of fiber crossings. When the tissue contains only parallel fibers, the corresponding susceptibility can be characterized by a rank-2 tensor; when the tissue contains multiple fiber orientations, the behavior of magnetic susceptibility no longer follows the sine-squared relationship and cannot be characterized by a single rank- 2 tensor. The orientation information provided by the magnetic susceptibility was consistent with that by HOT diffusion model. Susceptibility and diffusion together may provide a more complete characterization of white matter microstructure.

\section{ACKNOWLEDGMENTS}

The authors thank Arnaud Guidon for implementing the Hermite tensor function in the $\mathrm{C}$ language. The authors thank Ching-Po Lin, Ph.D., for providing the phantom diffusion data. The study was supported in part by the National Institutes of Health through grant R00EB007182. 


\section{REFERENCES}

Alexander, D. C., Barker, G. J., and Arridge, S. R. (2002). Detection and modeling of non-Gaussian apparent diffusion coefficient profiles in human brain data. Magn. Reson. Med. 48, 331-340.

Basser, P. J., and Pajevic, S. (2007). Spectral decomposition of a 4 thorder covariance tensor: applications to diffusion tensor MRI. Sig. Process. 87, 220-236.

Basser, P. J., Mattiello, J., and LeBihan, D. (1994). MR diffusion tensor spectroscopy and imaging. Biophys. J. 66, 259-267.

Basser, P. J., Pajevic, S., Pierpaoli, C., Duda, J., and Aldroubi, A. (2000). In vivo fiber tractography using DTMRI data. Magn. Reson. Med. 44, 625-632.

Basu, S., Fletcher, T., and Whitaker, R. (2006). Rician noise removal in diffusion tensor MRI. Med. Image Comput. Comput. Assist. Interv. 9(Pt 1), 117-125.

Beaulieu, C. (2002). The basis of anisotropic water diffusion in the nervous system - a technical review. NMR Biomed. 15, 435-455.

Browaeys, J. T., and Chevrot, S. (2004). Decomposition of the elastic tensor and geophysical applications. Geophys. J. Int. 159, 667-678.

Conturo, T. E., Lori, N. F., Cull, T. S., Akbudak, E., Snyder, A. Z., Shimony, J. S., et al. (1999). Tracking neuronal fiber pathways in the living human brain. Proc. Natl. Acad. Sci. U.S.A. 96, 10422-10427.

Duyn, J. H., van Gelderen, P., Li, T. Q., de Zwart, J. A., Koretsky, A. P., and Fukunaga, M. (2007). High-field MRI of brain cortical substructure based on signal phase. Proc. Natl. Acad. Sci. U.S.A. 104, 11796-11801.

Frank, L. R. (2001). Anisotropy in high angular resolution diffusionweighted MRI. Magn. Reson. Med. 45, 935-939.

Frank, L. R. (2002). Characterization of anisotropy in high angular resolution diffusion-weighted MRI. Magn. Reson. Med. 47, 1083-1099.

Jensen, J. H., Helpern, J. A., Ramani, A., Lu, H., and Kaczynski, K. (2005). Diffusional kurtosis imaging: the quantification of nongaussian water diffusion by means of magnetic resonance imaging. Magn. Reson. Med. 53, 1432-1440.
King, M. D., Houseman, J., Roussel, S. A., van Bruggen, N., Williams, S. R., and Gadian, D. G. (1994). q-space imaging of the brain. Magn. Reson. Med. 32, 707-713.

Lee, J., Shmueli, K., Fukunaga, M., van Gelderen, P., Merkle, H., Silva, A. C., et al. (2010). Sensitivity of MRI resonance frequency to the orientation of brain tissue microstructure. Proc. Natl. Acad. Sci. U.S.A. 107, 5130-5135.

Li, W., Wu, B., and Liu, C. (2011). Quantitative susceptibility mapping of human brain reflects spatial variation in tissue composition. Neuroimage 55, 1645-1656.

Li, W., Wu, B., Avram, A. V., and Liu, C. (2012a). Magnetic susceptibility anisotropy of human brain in vivo and its molecular underpinnings. Neuroimage 59, 2088-2097.

Li, X., Vikram, D. S., Lim, I. A., Jones, C. K., Farrell, J. A., and van Zijl, P. C. (2012b). Mapping magnetic susceptibility anisotropies of white matter in vivo in the human brain at 7T. Neuroimage 62, 314-330.

Lin, C. P., Wedeen, V. J., Chen, J. H., Yao, C., and Tseng, W. Y. (2003). Validation of diffusion spectrum magnetic resonance imaging with manganese-enhanced rat optic tracts and ex vivo phantoms. Neuroimage 19, 482-495.

Liu, C. (2010). Susceptibility tensor imaging. Magn. Reson. Med. 63 1471-1477.

Liu, C., and Li, W. (2013). Imaging neural architecture of the brain based on its multipole magnetic response. Neuroimage 67, 193-202.

Liu, C., Bammer, R., Acar, B., and Moseley, M. E. (2004). Characterizing non-Gaussian diffusion by using generalized diffusion tensors. Magn. Reson. Med. 51, 924-937.

Liu, C., Bammer, R., Acar, B., and Moseley, M. E. (2003a). "Generalized diffusion tensor imaging (GDTI) using higher order tensor (HOT) statistics," in Proceedings of the 11th Annual Meeting of ISMRM (Toronto, ON), 242.

Liu, C., Bammer, R., and Moseley, M. E. (2003b). Generalized diffusion tensor imaging (GDTI): a method for characterizing and imaging diffusion anisotropy caused by non-gaussian diffusion. Isr. J. Chem. 145-154.

Liu, C., Li, W., Johnson, G. A., and Wu, B. (2011). High-field (9.4 T) MRI of brain dysmyelination by quantitative mapping of magnetic susceptibility. Neuroimage 56, 930-938.

Liu, C., Li, W., Wu, B., Jiang, Y. and Johnson, G. A. (2012). 3D fiber tractography with susceptibility tensor imaging. Neuroimage 59, 1290-1298.

Miehe, C. (1993). Computation of isotropic tensor functions. Commun. Numer. Methods Eng. 9, 889-896.

Mori, S., Crain, B. J., Chacko, V. P., and van Zijl, P. C. (1999). Threedimensional tracking of axonal projections in the brain by magnetic resonance imaging. Ann. Neurol. 45, 265-269.

Moseley, M. E., Cohen, Y., Kucharczyk, J., Mintorovitch, J., Asgari, H. S. Wendland, M. F., et al. (1990). Diffusion-weighted MR imaging of anisotropic water diffusion in cat central nervous system. Radiology 176, 439-445.

Muti, D., and Bourennane, S. (2005). Multiway filtering based on fourthorder cumulants. EURASIP J. Appl. Sig. Process. 2005, 1147-1158.

Nemat-Nasser, S., and Hori, M. (1999). Micromechanics: Overall Properties of Heterogeneous Materials. Amsterdam; New York: Elsevier.

Ozarslan, E., and Mareci, T. H. (2003). Generalized diffusion tensor imaging and analytical relationships between diffusion tensor imaging and high angular resolution diffusion imaging. Magn. Reson. Med. 50, 955-965.

Paige, C. C., and Saunders, M. A (1982). Lsqr - an algorithm for sparse linear-equations and sparse least-squares. ACM Trans. Math. Softw. 8, 43-71.

Schweser, F., Sommer, K., Deistung, A., and Reichenbach, J. R. (2012). Quantitative susceptibility mapping for investigating subtle susceptibility variations in the human brain. Neuroimage 62, 2083-2100.

Sijbers, J., and den Dekker, A. J. (2004). Maximum likelihood estimation of signal amplitude and noise variance from MR data. Magn. Reson. Med. 51, 586-594.
Song, S. K., Sun, S. W., Ramsbottom, M. J., Chang, C., Russell, J., and Cross, A. H. (2002). Dysmyelination revealed through MRI as increased radial (but unchanged axial) diffusion of water. Neuroimage 17, 1429-1436.

Tuch, D. S. (2004). Q-ball imaging. Magn. Reson. Med. 52, 1358-1372.

Tuch, D. S., Reese, T. G., Wiegell, M. R., Makris, N., Belliveau, J. W., and Wedeen, V. J. (2002). High angular resolution diffusion imaging reveals intravoxel white matter fiber heterogeneity. Magn. Reson. Med. 48, 577-582.

Wedeen, V. J., Wang, R. P., Schmahmann, J. D., Benner, T., Tseng, W. Y., Dai, G., et al. (2008). Diffusion spectrum magnetic resonance imaging (DSI) tractography of crossing fibers. Neuroimage 41, 1267-1277.

Wu, B., Li, W., Guidon, A., and Liu, C. (2012). Whole brain susceptibility mapping using compressed sensing. Magn. Reson. Med. 67, 137-147.

Zucker, U., and Schulz, H. (1982). Statistical approaches for the treatment of anharmonic motion in crystals. I. A comparison of the most frequently used formalisms of anharmonic thermal vibrations. Acta Crystallogr. Sect. A 38, 563-568.

Conflict of Interest Statement: The authors declare that the research was conducted in the absence of any commercial or financial relationships that could be construed as a potential conflict of interest.

Received: 24 October 2012; paper pending published: 28 November 2012; accepted: 18 February 2013; published online: 06 March 2013

Citation: Liu C, Murphy NE and Li W (2013) Probing white-matter microstructure with higher-order diffusion tensors and susceptibility tensor MRI. Front. Integr. Neurosci. 7:11. doi: 10.3389/fnint.2013.00011

Copyright (C) 2013 Liu, Murphy and Li. This is an open-access article distributed under the terms of the Creative Commons Attribution License, which permits use, distribution and reproduction in other forums, provided the original authors and source are credited and subject to any copyright notices concerning any third-party graphics etc. 